\title{
A New Evaluation for Solving the Fully Fuzzy Data Envelopment Analysis with Z-Numbers
}

\author{
Ali Namakin, Seyyed Esmaeil Najafi* (D), Mohammad Fallah and Mehrdad Javadi \\ Department of Industrial Engineering, Science and Research Branch, Islamic Azad University, Tehran \\ 46818-53617, Iran; a_namakin@azad.ac.ir (A.N.); Mohammad.fallah43@yahoo.com (M.F.); \\ mjavadi@azad.ac.ir (M.J.) \\ * Correspondence: seyedesmailnajafi@gmail.com or najafi1515@yahoo.com
}

Received: 19 August 2018; Accepted: 29 August 2018; Published: 5 September 2018

\begin{abstract}
There are numerous models for solving the efficiency evaluation in data envelopment analysis (DEA) with fuzzy input and output data. However, because of the limitation of those strategies, they cannot be implemented for solving fully fuzzy DEA (FFDEA). Furthermore, in real-world problems with imprecise data, fuzziness is not sufficient to consider, and the reliability of the information is also very vital. To overcome these flaws, this paper presented a new method for solving the fully fuzzy DEA model where all parameters are Z-numbers. The new approach is primarily based on crisp linear programming and has a simple structure. Moreover, it is proved that the only existing method to solve FFDEA with Z-numbers is not valid. An example is also presented to illustrate the efficiency of our proposed method and provide an explanation for the content of the paper.
\end{abstract}

Keywords: data envelopment analysis; Z-numbers; full fuzzy environment; fuzzy efficiency

\section{Introduction}

Data envelopment analysis (DEA) is a linear programming method for measuring the relative efficiencies of homogeneous decision-making units (DMUs) without knowing production functions (i.e., just by utilizing input and output information) [1,2]. The DEA technique has just been effectively connected in various cases such as broadcasting companies, banking institutions, R\&D organizations, health care services, manufacturing, telecommunications, and supply chain management. Classical DEA models, such as CCR and BCC models [1,2], need crisp inputs and outputs, which are not typically accessible in real-world applications. However, the observed values of the input and output information in real-world issues are imprecise or vague [3-12] and imprecise evaluations could also be the end result of the unquantifiable, incomplete and non-available facts.

It is beneficial to consider the information of experts about the parameters as fuzzy data. The idea of the fuzzy set became established in [13]. After this, many researchers have applied this theory to different problems; see [14-22] and references therein. There are also many studies reported utilizing fuzzy set theory in DEA; see [23-33] and references therein.

Sengupta was the first to analyze DEA models in a fuzzy environment [23]. Triantis and Girod [24] combine DEA and fuzzy parametric programming to handle random measurement errors in input and output data. Kao and Liu [24] accompanied the simple concept of transforming a fuzzy DEA model to a crisp DEA model and presented an approach to measure the efficiencies of the DMUs. According to fuzzy arithmetic operations and fuzzy comparisons among fuzzy numbers, Dia [26] planned a model of fuzzy DEA (FDEA). Garcia et al. [27] applied the possibility of DEA version for failure mode and effects analysis (FMEA) and proposed a fuzzy DEA method to figure out ranking indices. Wang et al. [28] developed fuzzy DEA models with fuzzy inputs and outputs 
through fuzzy arithmetic. To obtain the efficiencies of DMUs as fuzzy numbers, they converted the proposed fuzzy DEA models into three linear programming (LP) models. Wang and Chin [29] utilized a fuzzy expected value approach and suggested the optimistic and the pessimistic efficiencies to solve FDEA. Emrouznejad et al. [30] presented a taxonomy of the FDEA methods and provided a classification scheme with six categories. Puri and Yadav [31] applied the $\alpha$-cut approach and provided a cross-efficiency technique for a fuzzy DEA model with undesirable fuzzy outputs. Wanke et al. [32] developed a new FDEA model and, using the bootstrap-truncated regressions, evaluated the efficiency of the Mozambican Banks. Hatami-Marbini et al. [33] proposed a comprehensive cross-efficiency fuzzy DEA approach for supplier evaluation. Readers can also refer to [34-43] for reviews of more recent research on fuzzy DEA approaches.

However, because of the limitations of the above methods, they cannot be implemented for solving fully fuzzy DEA (FFDEA), where all the inputs and outputs, as well as the decision variables, are fuzzy numbers. To the best of our knowledge, there is not much research on FFDEA. Hatami-Marbini et al. [44] utilized a fully fuzzy linear programming problem presented by an FFDEA model. Kazemi and Alimi [45] proposed an FFDEA model based on the ranking function. The feature of this proposed model is that it considers three situations for the problem and solves them simultaneously. Puri and Yadav [46] developed a new FFDEA model with undesirable factors and applied this model on multi-component FFDEA. Khaleghi et al. [47], based on simplex techniques and multi-objective optimization, obtain the fuzzy efficiency of FFDEA model.

Although the fuzzy set theory has been introduced as a powerful tool to quantify vague data, and several authors have suggested various fuzzy methods in DEA, there is a key inadequacy in past methodologies. A critical problem is that in classical fuzzy sets, the degree of sureness of information is not taken into account. When dealing with real information, fuzziness is not enough to take into account and the reliability of the information is vital. Recently, Sotoudeh-Anvari et al. [48] suggested a new FFDEA in a fuzzy situation with Z-numbers. The Z-number is a novel fuzzy notion which has more potential to articulate the vague circumstances of real applications. A Z-number has two components used to express a value of an arbitrary variable $X, Z=(A, B)$, where $A$ is an assessment of a value of $X$ and $B$ is an assessment of certainty of $A$ [49]. Sotoudeh-Anvari et al.'s model [48] is based on Kang et al.'s [50] and Allahviranloo et al.'s [51] methods and has low computational intricacy. However, the method has some flaws and is not valid.

In this paper, it is shown that the mentioned model is not true. To solve this drawback, we improved the model and propose a new algorithm. The rest of this work is ordered as follows: Section 2 presents some essential concepts regarding fuzzy set theory and converting a Z-number into a fuzzy number. In Section 3, we study the DEA, fuzzy DEA and fully fuzzified DEA models, briefly. Sotoudeh-Anvari et al.'s model [48] is reviewed in Section 4. Section 5 explains the shortcoming of the existing model [48]. The new FFDEA model with Z-numbers is also proposed in this section. A numerical example is given in Section 6. Finally, the paper is concluded.

\section{Preliminaries}

We start with some fundamental notations and starter results that we seek advice from later. For details, we refer to $[1,13,18,21,48-50]$.

\subsection{Definitions}

Definition 1 [18]. A fuzzy subset $\widetilde{A}$ of a set $X$ is defined by its membership function $\mu_{\widetilde{A}}: X \rightarrow[0,1]$, where the value of $\mu_{\widetilde{A}}(x)$ at $x$ shows the grade of membership of $x$ in $\widetilde{A}$. 
Definition 2 [18]. A triangular fuzzy number (TFNs) $\widetilde{A}$ can be defined by $(a, b, c)$, where $c \geq b \geq a$. The membership function $\mu_{\widetilde{A}}(x)$ is given by (1):

$$
\mu_{\widetilde{A}}(x)= \begin{cases}\frac{x-a}{b-a} & a \leq x \leq b \\ \frac{x-c}{b-c} & b \leq x \leq c \\ 0 & \text { otherwise }\end{cases}
$$

Definition 3 [21]. Let $\widetilde{A}=(a, b, c)$ be a triangular fuzzy number. Then $\widetilde{A}$ is called a non-negative fuzzy number if and only if $a \geq 0$.

Definition 4 [21]. Let $\widetilde{A}=(a, b, c)$ be a triangular fuzzy number. Then $\widetilde{A}$ is called an unrestricted fuzzy number if $a, b, c \in R$.

Definition 5 [21]. Consider $\widetilde{A}=(a, b, c)$ and $\widetilde{B}=(d, e, f)$ as two triangular fuzzy numbers, then we have:

(i) $\widetilde{A} \oplus \widetilde{B}=(a, b, c) \oplus(d, e, f)=(a+d, b+e, c+f)$,

(ii) $\widetilde{A}-\widetilde{B}=(a, b, c)-(d, e, f)=(a-f, b-e, c-d)$,

(iii) $\widetilde{A} \otimes \widetilde{B}=(\min (\gamma), b e, \max (\gamma))$ where, $\gamma=\{a d, a f, c d, c f\}$.

Definition 6 [21]. Consider $\widetilde{A}=(a, b, c)$ and $\widetilde{B}=(d, e, f)$ as two triangular fuzzy numbers. Then these numbers are equal if and only if $a=d, b=e$ and $c=f$.

Definition 7 [21]. Consider $\widetilde{A}=(a, b, c)$ as a triangular fuzzy number. Then the ranking function of $\widetilde{A}$ is defined as follows:

$$
R(\widetilde{A})=\frac{1}{4}(a+2 b+c)
$$

Definition 8 [21]. Suppose $\widetilde{A}$ and $\widetilde{B}$ be two triangular fuzzy numbers, then

(i) $\widetilde{A} \leq \widetilde{B}$ if and only if $R(\widetilde{A}) \leq R(\widetilde{B})$.

(ii) $\widetilde{A}<\widetilde{B}$ if and only if $R(\widetilde{A})<R(\widetilde{B})$.

Definition 9 [48]. A triangular fuzzy number can also be defined as $\widetilde{A}=(M, \alpha, \beta)$ which is referred to as a left right ( $L-R)$ fuzzy number. $M$ Is the central value, $\alpha$ is the left width (spread) and $\beta$ is the right width (spread). The membership function also has the following form:

$$
\mu_{\widetilde{A}}(x)= \begin{cases}\frac{x-M+\alpha}{\alpha} & M-\alpha \leq x \leq M \\ \frac{M-x+\beta}{\beta} & M \leq x \leq M+\beta \\ 0 & \text { otherwise }\end{cases}
$$

Remark 1. By Definitions 2 and 6 , we can see that a triangular fuzzy number $\widetilde{A}=(a, b, c)$ can be represented as $\widetilde{A}=(M, \alpha, \beta)$, where $a=M-\alpha, b=M$ and $c=M+\beta$.

So, based on Definitions 3-5, we have:

Definition 10. A $L-R$ fuzzy number $\widetilde{A}=(M, \alpha, \beta)$ is called a non-negative (positive) fuzzy number if and only if $M-\alpha \geq 0 \quad(M-\alpha>0)$. 
Definition 11. Let $\widetilde{A}=\left(M^{A}, \alpha^{A}, \beta^{A}\right)$ and $\widetilde{B}=\left(M^{B}, \alpha^{B}, \beta^{B}\right)$ be two triangular fuzzy numbers and $\lambda$ is a non-fuzzy number. Then we have:

$$
\begin{gathered}
\widetilde{A}+\widetilde{B}=\left(M^{A}, \alpha^{A}, \beta^{A}\right)+\left(M^{B}, \alpha^{B}, \beta^{B}\right)=\left(M^{A}+M^{B}, \alpha^{A}+\alpha^{B}, \beta^{A}+\beta^{B}\right) \\
\widetilde{A}-\widetilde{B}=\left(M^{A}, \alpha^{A}, \beta^{A}\right)-\left(M^{B}, \alpha^{B}, \beta^{B}\right)=\left(M^{A}-M^{B}, \alpha^{A}+\beta^{B}, \beta^{A}+\alpha^{B}\right) \\
\lambda \widetilde{A}=\left(\lambda M^{A}, \lambda \alpha^{A}, \lambda \beta^{A}\right) \quad \lambda>0
\end{gathered}
$$

And for non-negative fuzzy numbers $\widetilde{A}, \widetilde{B}$ the multiplication is defined as follows:

$$
\widetilde{A} \otimes \widetilde{B}=\left(M^{A} M^{B}, M^{A} \alpha^{B}+M^{B} \alpha^{A}-\alpha^{A} \alpha^{B}, M^{A} \beta^{B}+M^{B} \beta^{A}+\beta^{A} \beta^{B}\right)
$$

Definition 12. Let $\widetilde{A}=\left(M^{A}, \alpha^{A}, \beta^{A}\right)$ be any triangular fuzzy number, then the ranking function of $\widetilde{A}$ is as follows:

$$
R(\widetilde{A})=M^{A}+\frac{\beta^{A}-\alpha^{A}}{4} .
$$

Definition 13 [49]. A Z-number is an ordered pair of fuzzy numbers indicated as $Z=(\widetilde{A}, \widetilde{B})$. The first component, $\widetilde{A}$, is a fuzzy restriction and the second component, $\widetilde{B}$, is a level of reliability of the first component. For ease, $A$ and $B$ are supposed to be triangular fuzzy numbers.

\subsection{Converting a Z-Number into a Fuzzy Number}

In order to make more computations, the Z-number should be transformed into a usual fuzzy number. Kang et al. [50] presented an efficient and very easy to implement approach, called Kang et al.'s method, for turning a Z-number into a classical fuzzy number based on the fuzzy expectation. Kang et al.'s method is described as follows:

Suppose a Z-number is $Z=(\widetilde{A}, \widetilde{B})$.

Step 1: Convert the second part $(\widetilde{B})$ into a crisp number. Defuzzification transforms a fuzzy number into a crisp value. The most commonly used defuzzification technique is the centroid defuzzification approach. This calculation is completed by using Equation (6).

$$
\alpha=\frac{\int x \mu_{\widetilde{B}}(x) d x}{\int \mu_{\widetilde{B}}(x) d x}
$$

We noted that when $\widetilde{B}=\left(b_{1}, b_{2}, b_{3}\right)$, Equation (6) becomes as follows:

$$
\alpha=\frac{b_{1}+b_{2}+b_{3}}{3}
$$

Step 2: The weighted Z-number can be defined as:

$$
\widetilde{Z}^{\alpha}=\left\{\left(x, \mu_{\widetilde{A}^{\alpha}}\right) \mid \mu_{\widetilde{A}^{\alpha}}=\alpha \mu_{\widetilde{A}}(x), x \in[0,1]\right\}
$$

Step 3: By multiplying $\sqrt{\alpha}$, convert the weighted Z-number into the following classical fuzzy number:

$$
\widetilde{Z}^{\prime}=\sqrt{\alpha} \times \widetilde{A}^{\alpha}=(\sqrt{\alpha} \times a, \sqrt{\alpha} \times b, \sqrt{\alpha} \times c, \sqrt{\alpha} \times d)
$$

In this way, the Z-number is transformed into a conventional fuzzy number. 


\section{DEA, FDEA and FFDEA}

The efficiency of a DMU is established as the ratio of sum weighted output to sum weighted input, subjected to happen between one and zero.

Let $\mathrm{p}$-th DMU (DMUp) be under consideration, then the CCR model for the relative efficiency is as follows [1]:

$$
\begin{array}{ll}
\theta_{p}{ }^{*}= & \max \frac{\sum_{r=1}^{s} u_{r} y_{r p}}{\sum_{i=1}^{m} v_{i} x_{i p}} \\
\text { s.t. } & \\
& \frac{\sum_{r=1}^{s} u_{r} y_{r j}}{\sum_{i=1}^{m} v_{i} x_{i j}} \leq 1, \quad \forall j \\
& u_{r}, v_{i} \geq 0 \quad \forall r, i .
\end{array}
$$

In this model, each DMU (suppose that we have $n$ DMUs) uses $m$ inputs $x_{i j}(i=1,2, \ldots, m)$, to obtain $s$ outputs $y_{r j}(r=1,2, \ldots, s)$. Here $u_{r}(r=1,2, \ldots, s)$ and $v_{i}(i=1,2, \ldots, m)$ are the weights of the $i$ th input and $r$ th output. This fractional program is calculated for every DMU to find out its best input and output weights. To simplify the computation, the nonlinear program shown as (10) can be converted to a linear program (LP) and the model is called the CCR model:

$$
\begin{array}{ll} 
& \theta_{p}{ }^{*}=\max \sum_{r=1}^{s} u_{r} y_{r p} \\
\text { s.t }: & \\
& \sum_{i=1}^{m} v_{i} x_{i p}=1 \\
& \sum_{r=1}^{s} u_{r} y_{r j}-\sum_{i=1}^{m} v_{i} x_{i j} \leq 0, \quad \forall j \\
& u_{r}, v_{i} \geq 0 \quad \forall r, i .
\end{array}
$$

We solve Equation (11) $n$-times to work out the efficiency of $n$ DMUs. If $\theta_{p}{ }^{*}=1$, we say that the DMUp is efficient, otherwise it is inefficient.

Fuzzy DEA (FDEA) is a strong method for evaluating the efficiency of DMUs with imprecise information. The fuzzy CCR model is defined as follows:

$$
\begin{array}{ll}
\theta_{p}{ }^{*}= & \max \sum_{r=1}^{s} u_{r} \widetilde{y}_{r p} \\
\text { s.t : } \quad & \\
& \sum_{i=1}^{m} v_{i} \widetilde{x}_{i p}=1 \\
& \sum_{r=1}^{s} u_{r} \widetilde{y}_{r j}-\sum_{i=1}^{m} v_{i} \widetilde{x}_{i j} \leq 0, \quad \forall j \\
& u_{r}, v_{i} \geq 0 \quad \forall r, i .
\end{array}
$$

where, $\widetilde{x}_{i j}(i=1,2, \ldots, m)$ and $\widetilde{y}_{r j}(r=1,2, \ldots, s)$ are fuzzy inputs and fuzzy outputs for the $j$ th DMU $(\mathrm{DMU} j)$. 
If all input and output data and all parameters are characterized by fuzzy numbers, we call this problem a fully fuzzy DEA (FFDEA) with the following model:

$$
\begin{array}{ll}
\theta_{p}^{*}= & \max \sum_{r=1}^{s} \widetilde{u}_{r} \widetilde{y}_{r p} \\
\text { s.t : } \quad & \\
& \sum_{i=1}^{m} \widetilde{v}_{i} \widetilde{x}_{i p}=1 \\
& \sum_{r=1}^{s} \widetilde{u}_{r} \widetilde{y}_{r j}-\sum_{i=1}^{m} \widetilde{v}_{i} \widetilde{x}_{i j} \leq 0, \quad \forall j \\
& \widetilde{u}_{r}, \widetilde{v}_{i} \geq 0 \quad \forall r, i
\end{array}
$$

where $\widetilde{x}_{i j}=\left(x_{i j}^{M}, x_{i j}^{\alpha}, x_{i j}^{\beta}\right), \widetilde{y}_{i j}=\left(y_{i j}^{M}, y_{i j}^{\alpha}, y_{i j}^{\beta}\right)$ and their weights given by $\widetilde{u}_{r}=\left(u_{r}^{M}, u_{r}^{\alpha}, u_{r}^{\beta}\right), \widetilde{v}_{r}=$ $\left(v_{r}^{M}, v_{r}^{\alpha}, v_{r}^{\beta}\right)$. Using the Charnes and Cooper transformation, we have the following model:

$$
\begin{aligned}
& \max \quad \theta_{p}=\sum_{r=1}^{s}\left(u_{r}^{M}, u_{r}^{\alpha}, u_{r}^{\beta}\right) \otimes\left(y_{r p}^{M}, y_{r p}^{\alpha}, y_{r p}^{\beta}\right) \\
& \text { s.t. }\left(v_{r}^{M}, v_{r}^{\alpha}, v_{r}^{\beta}\right) \otimes\left(x_{i p}^{M}, y_{i p}^{\alpha}, y_{i p}^{\beta}\right)=(1,0,0) \\
& \sum_{r=1}^{s}\left(u_{r}^{M}, u_{r}^{\alpha}, u_{r}^{\beta}\right) \otimes\left(y_{r j}^{M}, y_{r j}^{\alpha}, y_{r j}^{\beta}\right)-\sum_{i=1}^{m}\left(u_{r}^{M}, u_{r}^{\alpha}, u_{r}^{\beta}\right) \otimes\left(x_{i j}^{M}, y_{i j}^{\alpha}, y_{i j}^{\beta}\right) \leq(0,0,0) \quad \forall j \\
& \left(u_{r}^{M}, u_{r}^{\alpha}, u_{r}^{\beta}\right),\left(v_{r}^{M}, v_{r}^{\alpha}, v_{r}^{\beta}\right) \geq 0 \quad \forall r, j
\end{aligned}
$$

\section{Sotoudeh-Anvari et al.'s Algorithm}

Sotoudeh-Anvari et al. [48] proposed a regular technique to extend the DEA to the fully fuzzy environment with Z-numbers. Their model is as follows:

\section{Method 1.}

Step 1: Consider the inputs and outputs of each DMU as well as their weights by using Z-numbers. Using the Charnes and Cooper transformation we have:

$$
\begin{array}{ll} 
& \theta_{p} \approx=\max \sum_{r=1}^{s} \widetilde{\widetilde{u}}_{r} \widetilde{\widetilde{y}}_{r p} \\
\text { s.t }: & \\
& \sum_{i=1}^{m} \widetilde{\widetilde{v}}_{i} \widetilde{\widetilde{x}}_{i p}=1 \\
& \sum_{r=1}^{s} \widetilde{\widetilde{u}}_{r} \widetilde{\widetilde{y}}_{r j}-\sum_{i=1}^{m} \widetilde{\widetilde{v}}_{i} \widetilde{\widetilde{x}}_{i j} \leq 0, \quad \forall j \\
& \widetilde{\widetilde{u}}_{r}, \widetilde{\widetilde{v}}_{i} \geq 0 \quad \forall r, i \quad \quad \forall
\end{array}
$$

where $\approx$ point out the Z-numbers. 
Step 2: Using the Kang et al. [50] model, convert Z-numbers into usual fuzzy numbers. Then the inputs and outputs of each DMU convert into $\widetilde{x}_{i j}=\left(x_{i j}^{M}, x_{i j}^{\alpha}, x_{i j}^{\beta}\right)$ and $\widetilde{y}_{i j}=\left(y_{i j}^{M}, y_{i j}^{\alpha}, y_{i j}^{\beta}\right)$. Furthermore, their weights will be $\widetilde{u}_{r}=\left(u_{r}^{M}, u_{r}^{\alpha}, u_{r}^{\beta}\right), \widetilde{v}_{r}=\left(v_{r}^{M}, v_{r}^{\alpha}, v_{r}^{\beta}\right)$ and we have:

$$
\begin{aligned}
& \max \quad \widetilde{\theta}_{p}=\sum_{r=1}^{s}\left(u_{r}^{M}, u_{r}^{\alpha}, u_{r}^{\beta}\right) \otimes\left(y_{r p}^{M}, y_{r p}^{\alpha}, y_{r p}^{\beta}\right) \\
& \text { s.t. } \\
& \sum_{i=1}^{m}\left(v_{i}^{M}, v_{i}^{\alpha}, v_{i}^{\beta}\right) \otimes\left(x_{i p}^{M}, y_{i p}^{\alpha}, y_{i p}^{\beta}\right) \approx(1,0,0) \\
& \sum_{r=1}^{s}\left(u_{r}^{M}, u_{r}^{\alpha}, u_{r}^{\beta}\right) \otimes\left(y_{r j}^{M}, y_{r j}^{\alpha}, y_{r j}^{\beta}\right)-\sum_{i=1}^{m}\left(u_{r}^{M}, u_{r}^{\alpha}, u_{r}^{\beta}\right) \otimes\left(x_{i j}^{M}, y_{i j}^{\alpha}, y_{i j}^{\beta}\right) \leq(0,0,0) \quad \forall j \\
& \left(u_{r}^{M}, u_{r}^{\alpha}, u_{r}^{\beta}\right),\left(v_{i}^{M}, v_{i}^{\alpha}, v_{i}^{\beta}\right) \geq 0 \quad \forall r, i .
\end{aligned}
$$

Step 3: The fuzzy DEA Equation (16) can be transformed into the following DEA model:

$$
\begin{aligned}
& \max \quad \widetilde{\theta}_{p}=\sum_{r=1}^{s}\left(u_{r}^{M}\left(y_{r p}^{M}+y_{r p}^{\beta}-y_{r p}^{\alpha}\right), u_{r}^{\beta} y_{r p}^{M}, u_{r}^{\alpha} y_{r p}^{M}\right) \\
& \text { s.t. } \\
& \sum_{i=1}^{M}\left(v_{i}^{M}\left(x_{i p}^{M}+x_{i p}^{\beta}-x_{i p}^{\alpha}\right), v_{i}^{\beta} x_{i p}^{M}, v_{i}^{\alpha} x_{i p}^{M}\right)=1, \\
& \sum_{r=1}^{s}\left(u_{r}^{M}\left(y_{r j}^{M}+y_{r j}^{\beta}-y_{r j}^{\alpha}\right), u_{r}^{\beta} y_{r j}^{M}, u_{r}^{\alpha} y_{r j}^{M}\right)-\sum_{i=1}^{m}\left(v_{i}^{M}\left(x_{i j}^{M}+x_{i j}^{\beta}-x_{i j}^{\alpha}\right), v_{i}^{\beta} x_{i j}^{M}, v_{i}^{\alpha} x_{i j}^{M}\right) \leq(0,0,0) \quad \forall j \\
& \left(u_{r}^{M}, u_{r}^{\alpha}, u_{r}^{\beta}\right),\left(v_{i}^{M}, v_{i}^{\alpha}, v_{i}^{\beta}\right) \geq 0 \quad \forall r, i .
\end{aligned}
$$

Step 4: Convert the fuzzy DEA Equation (17) into the following LP model:

$$
\begin{gathered}
\max \quad \theta_{P}=R\left(\widetilde{\theta}_{P}\right)=\sum_{r=1}^{s}\left[u_{r}^{M}\left(y_{r p}^{M}+\left(\frac{1}{4}\right) y_{r p}^{\beta}-\left(\frac{1}{4}\right) y_{r p}^{\alpha}\right)+u_{r}^{\beta}\left(\left(\frac{1}{4}\right) y_{r p}^{M}\right)-u_{r}^{\alpha}\left(\left(\frac{1}{4}\right) y_{r p}^{M}\right)\right] \\
\text { s.t. } \\
\sum_{i=1}^{m}\left[v_{i}^{M}\left(x_{i p}^{M}+\left(\frac{1}{4}\right) x_{i p}^{\beta}-\left(\frac{1}{4}\right) x_{i p}^{\alpha}\right)+v_{i}^{\beta}\left(\left(\frac{1}{4}\right) x_{i p}^{M}\right)-v_{i}^{\alpha}\left(\left(\frac{1}{4}\right) x_{i p}^{M}\right)\right]=1 \\
\sum_{r=1}^{s}\left[u_{r}^{M}\left(y_{r j}^{M}+\left(\frac{1}{4}\right) y_{r j}^{\beta}-\left(\frac{1}{4}\right) y_{r j}^{\alpha}\right)\right]+u_{r}^{\beta}\left(\left(\frac{1}{4}\right) y_{r j}^{M}\right)-u_{r}^{\alpha}\left(\left(\frac{1}{4}\right) y_{r j}^{M}\right) \leq \\
\sum_{r=1}^{s}\left[v_{i}^{M}\left(x_{i j}^{M}+\left(\frac{1}{4}\right) x_{i j}^{\beta}-\left(\frac{1}{4}\right) x_{i j}^{\alpha}\right)+v_{i}^{\beta}\left(\left(\frac{1}{4}\right) x_{i j}^{M}\right)-v_{i}^{\alpha}\left(\left(\frac{1}{4}\right) x_{i j}^{M}\right)\right], \quad \forall j \\
u_{r}^{M}-u_{r}^{\alpha} \geq 0, \quad \forall r \\
u_{r}^{M}-\left(\frac{1}{4}\right) u_{r}^{\alpha}+\left(\frac{1}{4}\right) u_{r}^{\beta} \geq 0 \quad \forall r \\
v_{i}^{M}-u_{i}^{\alpha} \geq 0, \quad \forall i \\
v_{i}^{M}-\left(\frac{1}{4}\right) v_{i}^{\alpha}+\left(\frac{1}{4}\right) v_{i}^{\beta} \geq 0 \quad \forall i \\
u_{r}^{\alpha} \geq 0, u_{r}^{\beta} \geq 0, \quad \forall r \\
v_{i}^{\alpha} \geq 0, v_{i}^{\beta} \geq 0, \quad \forall i .
\end{gathered}
$$

Step 5: Run Equation (18) and obtain the optimal solutions of $u_{r}^{M^{*}}, u_{r}^{\alpha^{*}}, u_{r}^{\beta^{*}}, v_{i}^{M^{*}}, v_{i}^{\alpha^{*}}$ and $v_{i}^{\beta^{*}}$.

\section{Main Results}

In this section, we explain the shortcomings of Sotoudeh-Anvari et al.'s Algorithm [48] and present the new Algorithm. 


\subsection{The Shortcoming of the Existing Algorithm}

From Definition 11, if $\widetilde{A}=\left(M^{A}, \alpha^{A}, \beta^{A}\right)$ and $\widetilde{B}=\left(M^{B}, \alpha^{B}, \beta^{B}\right)$ are two non-negative triangular fuzzy numbers then:

$$
\widetilde{A} \otimes \widetilde{B}=\left(M^{A} M^{B}, M^{A} \alpha^{B}+M^{B} \alpha^{A}-\alpha^{A} \alpha^{B}, M^{A} \beta^{B}+M^{B} \beta^{A}+\beta^{A} \beta^{B}\right) .
$$

Nonetheless, it is evident from Step 3 of Method 1 that Sotoudeh-Anvari et al. [38], have utilized the wrong product:

$$
\widetilde{A} \otimes \widetilde{B}=\left(M^{A}\left(M^{B}+\beta^{B}-\alpha^{B}\right), \beta^{A} M^{B}, \alpha^{A} M^{B}\right),
$$

to transform the fuzzy DEA Equation (16) into the fuzzy DEA Equation (17). Henceforth, Method 1, proposed by Sotoudeh-Anvari et al. [48], is not substantial in its present frame.

\subsection{Improvement Model for FFDEA with Z-Numbers}

In this section, to remove the mentioned shortcoming, we proposed an improved model for fully fuzzy DEA with Z-numbers.

\section{Method 2.}

Step 1: Consider the DEA model that the inputs and outputs of each DMU as well as their weights are Z-numbers.

Step 2: Using Kang et al.'s [50] model, convert Z-numbers into usual fuzzy numbers and obtain a fully fuzzy DEA model with triangular fuzzy numbers.

Step 3: Using Definition 11, the fully fuzzy DEA model of Step 2 can be transformed into the following model:

$$
\begin{aligned}
& \max \widetilde{\theta}_{p}=\sum_{r=1}^{s}\left(u_{r}^{M} y_{r p}^{M}, u_{r}^{M} y_{r p}^{\alpha}+y_{r p}^{M} u_{r}^{\alpha}-u_{r}^{\alpha} y_{r p}^{\alpha}, u_{r}^{M} y_{r p}^{\beta}+y_{r p}^{M} u_{r}^{\beta}+u_{r}^{\beta} y_{r p}^{\beta}\right) \\
& \text { s.t. } \\
& \sum_{i=1}^{m}\left(v_{i}^{M} x_{i p}^{M}, v_{i}^{M} x_{i p}^{\alpha}+x_{i p}^{M} v_{i}^{\alpha}-v_{i}^{\alpha} x_{i p}^{\alpha}, v_{i}^{M} x_{i p}^{\beta}+x_{i p}^{M} v_{i}^{\beta}+v_{i}^{\beta} x_{i p}^{\beta}\right) \approx 1, \\
& \sum_{r=1}^{s}\left(u_{r}^{M} y_{r j}^{M}, u_{r}^{M} y_{r j}^{\alpha}+y_{r j}^{M} u_{r}^{\alpha}-u_{r}^{\alpha} y_{r j}^{\alpha}, u_{r}^{M} y_{r j}^{\beta}+y_{r j}^{M} u_{r}^{\beta}+u_{r}^{\beta} y_{r j}^{\beta}\right) \leq \\
& \sum_{i=1}^{m}\left(v_{i}^{M} x_{i j}^{M}, v_{i}^{M} x_{i j}^{\alpha}+x_{i j}^{M} v_{i}^{\alpha}-v_{i}^{\alpha} x_{i j}^{\alpha}, v_{i}^{M} x_{i j}^{\beta}+x_{i j}^{M} v_{i}^{\beta}+v_{i}^{\beta} x_{i j}^{\beta}\right) \quad \forall j \\
& \left(u_{r}^{M}, u_{r}^{\alpha}, u_{r}^{\beta}\right),\left(v_{i}^{M}, v_{i}^{\alpha}, v_{i}^{\beta}\right) \geq 0 \quad \forall r, i
\end{aligned}
$$

Step 4: Based on Definitions 7 and 8, convert the fuzzy DEA Equation (19) into the following model:

$$
\begin{aligned}
& \max \quad \theta_{P}=R\left(\widetilde{\theta}_{P}\right)=\sum_{r=1}^{s} R\left(\left(u_{r}^{M} y_{r p}^{M}, u_{r}^{M} y_{r p}^{\alpha}+y_{r p}^{M} u_{r}^{\alpha}-u_{r}^{\alpha} y_{r p}^{\alpha}, u_{r}^{M} y_{r p}^{\beta}+y_{r p}^{M} u_{r}^{\beta}+u_{r}^{\beta} y_{r p}^{\beta}\right)\right) \\
& \sum_{i=1}^{m} R\left(\left(v_{i}^{M} x_{i p}^{M}, v_{i}^{M} x_{i p}^{\alpha}+x_{i p}^{M} v_{i}^{\alpha}-v_{i}^{\alpha} x_{i p}^{\alpha}, v_{i}^{M} x_{i p}^{\beta}+x_{i p}^{M} v_{i}^{\beta}+v_{i}^{\beta} x_{i p}^{\beta}\right)\right)=R(1,1,1), \\
& \sum_{r=1}^{s} R\left(\left(u_{r}^{M} y_{r j}^{M}, u_{r}^{M} y_{r j}^{\alpha}+y_{r j}^{M} u_{r}^{\alpha}-u_{r}^{\alpha} y_{r j}^{\alpha}, u_{r}^{M} y_{r j}^{\beta}+y_{r j}^{M} u_{r}^{\beta}+u_{r}^{\beta} y_{r j}^{\beta}\right)\right) \leq \\
& \quad \sum_{i=1}^{m} R\left(\left(v_{i}^{M} x_{i j}^{M}, v_{i}^{M} x_{i j}^{\alpha}+x_{i j}^{M} v_{i}^{\alpha}-v_{i}^{\alpha} x_{i j}^{\alpha}, v_{i}^{M} x_{i j}^{\beta}+x_{i j}^{M} v_{i}^{\beta}+v_{i}^{\beta} x_{i j}^{\beta}\right)\right) \quad \forall j \\
& R\left(u_{r}^{M}, u_{r}^{\alpha}, u_{r}^{\beta}\right) \geq(0,0,0), R\left(v_{i}^{M}, v_{i}^{\alpha}, v_{i}^{\beta}\right) \geq(0,0,0), \quad \forall r, i \\
& u_{r}^{M}-u_{r}^{\alpha} \geq 0, \quad \forall r, \\
& v_{i}^{M}-v_{i}^{\alpha} \geq 0, \quad \forall i, \\
& u_{r}^{\alpha} \geq 0, u_{r}^{\beta} \geq 0, \quad \forall r \\
& v_{i}^{\alpha} \geq 0, v_{i}^{\beta} \geq 0, \quad \forall i .
\end{aligned}
$$


Step 5: Based on Definition 12, convert Equation (20) into the following model:

$$
\begin{aligned}
& \max \quad \theta_{P}=R\left(\widetilde{\theta}_{P}\right)=\sum_{r=1}^{s}\left[u_{r}^{M} y_{r p}^{M}+\frac{1}{4}\left[u_{r}^{M} y_{r p}^{\beta}+y_{r p}^{M} u_{r}^{\beta}+u_{r}^{\beta} y_{r p}^{\beta}\right]-\frac{1}{4}\left[u_{r}^{M} y_{r p}^{\alpha}+y_{r p}^{M} u_{r}^{\alpha}-u_{r}^{\alpha} y_{r p}^{\alpha}\right]\right] \\
& \text { s.t. } \\
& \sum_{i=1}^{m}\left[v_{i}^{M} x_{i p}^{M}+\frac{1}{4}\left[v_{i}^{M} x_{i p}^{\beta}+x_{i p}^{M} v_{i}^{\beta}+v_{i}^{\beta} x_{i p}^{\beta}\right]-\frac{1}{4}\left[v_{i}^{M} x_{i p}^{\alpha}+x_{i p}^{M} v_{i}^{\alpha}-v_{i}^{\alpha} x_{i p}^{\alpha}\right]\right]=1, \\
& \sum_{r=1}^{s}\left[u_{r}^{M} y_{r j}^{M}+\frac{1}{4}\left[u_{r}^{M} y_{r j}^{\beta}+y_{r j}^{M} u_{r}^{\beta}+u_{r}^{\beta} y_{r j}^{\beta}\right]-\frac{1}{4}\left[u_{r}^{M} y_{r j}^{\alpha}+y_{r j}^{M} u_{r}^{\alpha}-u_{r}^{\alpha} y_{r j}^{\alpha}\right]\right] \leq \\
& \sum_{i=1}^{m}\left[v_{i}^{M} x_{i j}^{M}+\frac{1}{4}\left[v_{i}^{M} x_{i j}^{\beta}+x_{i j}^{M} v_{i}^{\beta}+v_{i}^{\beta} x_{i j}^{\beta}\right]-\frac{1}{4}\left[v_{i}^{M} x_{i j}^{\alpha}+x_{i j}^{M} v_{i}^{\alpha}-v_{i}^{\alpha} x_{i j}^{\alpha}\right], \quad \forall j\right. \\
& u_{r}^{M}-u_{r}^{\alpha} \geq 0, \quad \forall r \\
& u_{r}^{M}-\left(\frac{1}{4}\right) u_{r}^{\alpha}+\left(\frac{1}{4}\right) u_{r}^{\beta} \geq 0 \quad \forall r \\
& v_{i}^{M}-u_{i}^{\alpha} \geq 0, \quad \forall i \\
& v_{i}^{M}-\left(\frac{1}{4}\right) v_{i}^{\alpha}+\left(\frac{1}{4}\right) v_{i}^{\beta} \geq 0 \quad \forall i \\
& u_{r}^{\alpha} \geq 0, u_{r}^{\beta} \geq 0, \quad \forall r \\
& v_{i}^{\alpha} \geq 0, v_{i}^{\beta} \geq 0, \quad \forall i .
\end{aligned}
$$

Step 6: Obtain the optimal solutions of $u_{r}^{M^{*}}, u_{r}^{\alpha^{*}}, u_{r}^{\beta^{*}}, v_{i}^{M^{*}}, v_{i}^{\alpha^{*}}$ and $v_{i}^{\beta^{*}}$.

\section{Numerical Example}

Sotoudeh-Anvari et al. [48], tackled the existing problem taken from Guo and Tanaka [52] to represent their proposed approach. However, as discussed in Section 5, there are some shortcomings in Method 1. Therefore, the results of Sotoudeh-Anvari et al. [48] are likewise not correct. In this section, the correct consequences of this problem are solved by Method 2.

Problem 1. Consider five DMUs with two inputs and two outputs where all the input and output data are designed as Z-numbers (see Table 1).

Table 1. Five decision-making units (DMUs) with two Z-number inputs and two Z-number outputs. $\mathrm{M}=$ medium $. \mathrm{MH}=$ medium high $\mathrm{H}=$ high $. \mathrm{VH}=$ very high.

\begin{tabular}{ccccc}
\hline DMU & Inputs 1 & Inputs 2 & Outputs 1 & Outputs 2 \\
\hline A & $((4.51,5.16,5.80), \mathrm{MH})$ & $((2.125,2.348,2.572), \mathrm{VH})$ & $((2.870,3.110,3.349), \mathrm{H})$ & $((4.545,4.904,5.263), \mathrm{H})$ \\
$\mathrm{B}$ & $((3.46,3.46,3.46), \mathrm{H})$ & $((1.674,1.794,1.913), \mathrm{H})$ & $((2.460,2.460,2.460), \mathrm{VH})$ & $((4.263,4.521,4.780), \mathrm{MH})$ \\
$\mathrm{C}$ & $((4.92,5.48,6.04), \mathrm{VH})$ & $((2.631,3.11,3.588), \mathrm{H})$ & $((3.229,3.827,4.425), \mathrm{H})$ & $((4.809,5.704,6.599), \mathrm{VH})$ \\
$\mathrm{D}$ & $((4.80,5.80,6.79), \mathrm{M})$ & $((2.460,2.572,2.684), \mathrm{VH})$ & $((2.971,3.229,3.746), \mathrm{MH})$ & $((7.779,8.062,8.345), \mathrm{M})$ \\
$\mathrm{E}$ & $((7.05,7.77,8.49), \mathrm{H})$ & $((4.647,5.293,5.938), \mathrm{MH})$ & $((6.223,7.213,8.203), \mathrm{M})$ & $((7.775,8.851,9.928), \mathrm{H})$ \\
\hline
\end{tabular}

In Table 1, the linguistic variables need to be transformed into triangular fuzzy numbers which are listed in Table 2.

Table 2. Linguistic variables for measuring of the reliability of Z-numbers.

\begin{tabular}{ccc}
\hline Linguistic Term & Abbreviation & Corresponding TFNs \\
\hline Very Low & VL & $(0.1,0.2,0.3)$ \\
Low & L & $(0.2,0.3,0.4)$ \\
Medium Low & ML & $(0.3,0.4,0.5)$ \\
Medium & M & $(0.4,0.5,0.6)$ \\
Medium High & MH & $(0.5,0.6,0.7)$ \\
High & H & $(0.6,0.7,0.8)$ \\
Very High & VH & $(0.7,0.8,0.9)$ \\
\hline
\end{tabular}

By using Kang et al.'s [50] model, the Z-number values of Table 1 are converted into fuzzy numbers, which are listed in Table 3. 
Table 3. Data transformation from Z-numbers into fuzzy numbers.

\begin{tabular}{ccccc}
\hline DMU & Inputs 1 & Inputs 2 & Outputs 1 & Outputs 2 \\
\hline A & $(4,0.5,0.5)$ & $(2.1,0.2,0.2)$ & $(2.6,0.2,0.2)$ & $(4.1,0.3,0.3)$ \\
B & $(2.9,0,0)$ & $(1.5,0.1,0.1)$ & $(2.2,0,0)$ & $(3.5,0.2,0.2)$ \\
C & $(4.9,0.5,0.5)$ & $(2.6,0.4,0.4)$ & $(3.2,0.5,0.5)$ & $(5.1,0.8,0.8)$ \\
D & $(4.1,0.7,0.7)$ & $(2.3,0.1,0.1)$ & $(2.5,0.2,0.4)$ & $(5.7,0.2,0.2)$ \\
E & $(6.1,0.2,1)$ & $(4.1,0.5,0.5)$ & $(5.1,0.7,0.7)$ & $(7.4,0.9,0.9)$ \\
\hline
\end{tabular}

Now, we use Method 2 to solve the performance assessment problem. For example, Method 2 for $\mathrm{DMU}_{\mathrm{A}}$ can be used as follows:

Step 1: Obtain a fully fuzzy DEA model with triangular fuzzy numbers:

$$
\begin{aligned}
& \max \quad \widetilde{\theta}_{A} \approx(2.6,0.2,0.2) \otimes\left(u_{1}^{M}, u_{1}^{\alpha}, u_{1}^{\beta}\right)+(4.1,0.3,0.3) \otimes\left(u_{2}^{M}, u_{2}^{\alpha}, u_{2}^{\beta}\right) \\
& \text { s.t. } \\
& (4,0.5,0.5) \otimes\left(v_{1}^{M}, v_{1}^{\alpha}, v_{1}^{\beta}\right)+(2.1,0.2,0.2) \otimes\left(v_{2}^{M}, v_{2}^{\alpha}, v_{2}^{\beta}\right) \approx 1, \\
& {\left[(2.6,0.2,0.2) \otimes\left(u_{1}^{M}, u_{1}^{\alpha}, u_{1}^{\beta}\right)+(4.1,0.3,0.3) \otimes\left(u_{2}^{M}, u_{2}^{\alpha}, u_{2}^{\beta}\right)\right]-} \\
& {\left[(4,0.5,0.5) \otimes\left(v_{1}^{M}, v_{1}^{\alpha}, v_{1}^{\beta}\right)+(2.1,0.2,0.2) \otimes\left(v_{2}^{M}, v_{2}^{\alpha}, v_{2}^{\beta}\right)\right] \leq(0,0,0),} \\
& {\left[(2.2,0,0) \otimes\left(u_{1}^{M}, u_{1}^{\alpha}, u_{1}^{\beta}\right)+(3.5,0.2,0.2) \otimes\left(u_{2}^{M}, u_{2}^{\alpha}, u_{2}^{\beta}\right)\right]-} \\
& {\left[(2.9,0,0) \otimes\left(v_{1}^{M}, v_{1}^{\alpha}, v_{1}^{\beta}\right)+(1.5,0.1,0.1) \otimes\left(v_{2}^{M}, v_{2}^{\alpha}, v_{2}^{\beta}\right)\right] \leq(0,0,0),} \\
& {\left[(3.2,0.5,0.5) \otimes\left(u_{1}^{M}, u_{1}^{\alpha}, u_{1}^{\beta}\right)+(5.1,0.8,0.8) \otimes\left(u_{2}^{M}, u_{2}^{\alpha}, u_{2}^{\beta}\right)\right]-} \\
& {\left[(4.9,0.5,0.5) \otimes\left(v_{1}^{M}, v_{1}^{\alpha}, v_{1}^{\beta}\right)+(2.6,0.4,0.4) \otimes\left(v_{2}^{M}, v_{2}^{\alpha}, v_{2}^{\beta}\right)\right] \leq(0,0,0),} \\
& {\left[(2.5,0.2,0.4) \otimes\left(u_{1}^{M}, u_{1}^{\alpha}, u_{1}^{\beta}\right)+(5.7,0.9,0.9) \otimes\left(u_{2}^{M}, u_{2}^{\alpha}, u_{2}^{\beta}\right)\right]-} \\
& {\left[(4.1,0.7,0.7) \otimes\left(v_{1}^{M}, v_{1}^{\alpha}, v_{1}^{\beta}\right)+(2.3,0.1,0.1) \otimes\left(v_{2}^{M}, v_{2}^{\alpha}, v_{2}^{\beta}\right)\right] \leq(0,0,0),} \\
& {\left[(5.1,0.7,0.7) \otimes\left(u_{1}^{M}, u_{1}^{\alpha}, u_{1}^{\beta}\right)+(7.4,0.9,0.9) \otimes\left(u_{2}^{M}, u_{2}^{\alpha}, u_{2}^{\beta}\right)\right]-} \\
& {\left[(6.1,0.2,1) \otimes\left(v_{1}^{M}, v_{1}^{\alpha}, v_{1}^{\beta}\right)+(4.1,0.5,0.5) \otimes\left(v_{2}^{M}, v_{2}^{\alpha}, v_{2}^{\beta}\right)\right] \leq(0,0,0),} \\
& \left(u_{r}^{M}, u_{r}^{\alpha}, u_{r}^{\beta}\right),\left(v_{i}^{M}, v_{i}^{\alpha}, v_{i}^{\beta}\right) \geq 0 \quad \forall r, i
\end{aligned}
$$

Step 2: Using the Definition 11, the fully fuzzy DEA model of Step 2 can be transformed into the following model:

$$
\begin{aligned}
& \max \quad \widetilde{\theta}_{A} \approx\left(2.6 u_{1}^{M}, 0.2 u_{1}^{M}+2.4 u_{1}^{\alpha}, 0.2 u_{1}^{M}+2.8 u_{1}^{\beta}\right)+\left(4.1 u_{2}^{M}, 0.3 u_{2}^{M}+3.8 u_{2}^{\alpha}, 0.3 u_{2}^{M}+4.4 u_{2}^{\beta}\right) \\
& \text { s.t. } \\
& \left(4 v_{1}^{M}, 0.5 v_{1}^{M}+3.5 v_{1}^{\alpha}, 0.5 v_{1}^{M}+4.5 v_{1}^{\beta}\right)+\left(2.1 v_{2}^{M}, 0.2 v_{2}^{M}+1.9 v_{2}^{\alpha}, 0.2 v_{2}^{M}+2.3 v_{2}^{\beta}\right) \approx(1,1,1), \\
& \left(2.6 u_{1}^{M}, 0.2 u_{1}^{M}+2.4 u_{1}^{\alpha}, 0.2 u_{1}^{M}+2.8 u_{1}^{\beta}\right)+\left(4.1 u_{2}^{M}, 0.3 u_{2}^{M}+3.8 u_{2}^{\alpha}, 0.3 u_{2}^{M}+4.4 u_{2}^{\beta}\right) \\
& -\left(4 v_{1}^{M}, 0.5 v_{1}^{M}+3.5 v_{1}^{\alpha}, 0.5 v_{1}^{M}+4.5 v_{1}^{\beta}\right)-\left(2.1 v_{2}^{M}, 0.2 v_{2}^{M}+1.9 v_{2}^{\alpha}, 0.2 v_{2}^{M}+2.3 v_{2}^{\beta}\right) \leq(0,0,0), \\
& \left(2.2 u_{1}^{M}, 2.2 u_{1}^{\alpha}, 2.2 u_{1}^{\beta}\right)+\left(3.5 u_{2}^{M}, 0.2 u_{2}^{M}+3.3 u_{2}^{\alpha}, 0.2 u_{2}^{M}+3.7 u_{2}^{\beta}\right) \\
& -\left(2.9 v_{1}^{M}, 2.9 v_{1}^{\alpha}, 2.9 v_{1}^{\beta}\right)-\left(1.5 v_{2}^{M}, 0.1 v_{2}^{M}+1.4 v_{2}^{\alpha}, 0.1 v_{2}^{M}+1.6 v_{2}^{\beta}\right) \leq(0,0,0), \\
& \left(3.2 u_{1}^{M}, 0.5 u_{1}^{M}+2.7 u_{1}^{\alpha}, 0.5 u_{1}^{M}+3.7 u_{1}^{\beta}\right)+\left(5.1 u_{2}^{M}, 0.8 u_{2}^{M}+4.3 u_{2}^{\alpha}, 0.8 u_{2}^{M}+5.9 u_{2}^{\beta}\right) \\
& -\left(4.9 v_{1}^{M}, 0.5 v_{1}^{M}+4.4 v_{1}^{\alpha}, 0.5 v_{1}^{M}+5.4 v_{1}^{\beta}\right)-\left(2.6 v_{2}^{M}, 0.4 v_{2}^{M}+2.2 v_{2}^{\alpha}, 0.4 v_{2}^{M}+3 v_{2}^{\beta}\right) \leq(0,0,0), \\
& \left(2.5 u_{1}^{M}, 0.2 u_{1}^{M}+2.3 u_{1}^{\alpha}, 0.4 u_{1}^{M}+2.9 u_{1}^{\beta}\right)+\left(5.7 u_{2}^{M}, 0.2 u_{2}^{M}+5.5 u_{2}^{\alpha}, 0.2 u_{2}^{M}+5.9 u_{2}^{\beta}\right) \\
& -\left(4.1 v_{1}^{M}, 0.7 v_{1}^{M}+3.4 v_{1}^{\alpha}, 0.7 v_{1}^{M}+4.8 v_{1}^{\beta}\right)-\left(2.3 v_{2}^{M}, 0.1 v_{2}^{M}+2.2 v_{2}^{\alpha}, 0.1 v_{2}^{M}+2.4 v_{2}^{\beta}\right) \leq(0,0,0), \\
& \left(5.1 u_{1}^{M}, 0.7 u_{1}^{M}+4.4 u_{1}^{\alpha}, 0.7 u_{1}^{M}+5.8 u_{1}^{\beta}\right)+\left(7.4 u_{2}^{M}, 0.9 u_{2}^{M}+6.5 u_{2}^{\alpha}, 0.9 u_{2}^{M}+8.3 u_{2}^{\beta}\right) \\
& -\left(6.1 v_{1}^{M}, 0.2 v_{1}^{M}+5.9 v_{1}^{\alpha}, v_{1}^{M}+7.1 v_{1}^{\beta}\right)-\left(4.1 v_{2}^{M}, 0.5 v_{2}^{M}+3.6 v_{2}^{\alpha}, 0.5 v_{2}^{M}+4.6 v_{2}^{\beta}\right) \leq(0,0,0), \\
& \left(u_{r}^{M}, u_{r}^{\alpha}, u_{r}^{\beta}\right),\left(v_{i}^{M}, v_{i}^{\alpha}, v_{i}^{\beta}\right) \geq(0,0,0) \quad \forall r, i
\end{aligned}
$$


Step 3: Based on Definitions 7 and 8, convert the above fuzzy DEA model to the following model:

$$
\begin{aligned}
& \max \quad \theta_{A}=R\left(\widetilde{\theta}_{A}\right)=R\left(\left(2.6 u_{1}^{M}, 0.2 u_{1}^{M}+2.4 u_{1}^{\alpha}, 0.2 u_{1}^{M}+2.8 u_{1}^{\beta}\right)+\left(4.1 u_{2}^{M}, 0.3 u_{2}^{M}+3.8 u_{2}^{\alpha}, 0.3 u_{2}^{M}+4.4 u_{2}^{\beta}\right)\right) \\
& \begin{array}{l}
\text { s.t. } \\
R\left(\left(4 v_{1}^{M}, 0.5 v_{1}^{M}+3.5 v_{1}^{\alpha}, 0.5 v_{1}^{M}+4.5 v_{1}^{\beta}\right)+\left(2.1 v_{2}^{M}, 0.2 v_{2}^{M}+1.9 v_{2}^{\alpha}, 0.2 v_{2}^{M}+2.3 v_{2}^{\beta}\right)\right)=R(1,1,1),
\end{array} \\
& R\left(\begin{array}{c}
\left(2.6 u_{1}^{M}, 0.2 u_{1}^{M}+2.4 u_{1}^{\alpha}, 0.2 u_{1}^{M}+2.8 u_{1}^{\beta}\right)+\left(4.1 u_{2}^{M}, 0.3 u_{2}^{M}+3.8 u_{2}^{\alpha}, 0.3 u_{2}^{M}+4.4 u_{2}^{\beta}\right) \\
-\left(4 v_{1}^{M}, 0.5 v_{1}^{M}+3.5 v_{1}^{\alpha}, 0.5 v_{1}^{M}+4.5 v_{1}^{\beta}\right)-\left(2.1 v_{2}^{M}, 0.2 v_{2}^{M}+1.9 v_{2}^{\alpha}, 0.2 v_{2}^{M}+2.3 v_{2}^{\beta}\right)
\end{array}\right) \leq R(0,0,0), \\
& R\left(\begin{array}{c}
\left(2.2 u_{1}^{M}, 2.2 u_{1}^{\alpha}, 2.2 u_{1}^{\beta}\right)+\left(3.5 u_{2}^{M}, 0.2 u_{2}^{M}+3.3 u_{2}^{\alpha}, 0.2 u_{2}^{M}+3.7 u_{2}^{\beta}\right) \\
-\left(2.9 v_{1}^{M}, 2.9 v_{1}^{\alpha}, 2.9 v_{1}^{\beta}\right)-\left(1.5 v_{2}^{M}, 0.1 v_{2}^{M}+1.4 v_{2}^{\alpha}, 0.1 v_{2}^{M}+1.6 v_{2}^{\beta}\right)
\end{array}\right) \leq R(0,0,0), \\
& R\left(\begin{array}{c}
\left(3.2 u_{1}^{M}, 0.5 u_{1}^{M}+2.7 u_{1}^{\alpha}, 0.5 u_{1}^{M}+3.7 u_{1}^{\beta}\right)+\left(5.1 u_{2}^{M}, 0.8 u_{2}^{M}+4.3 u_{2}^{\alpha}, 0.8 u_{2}^{M}+5.9 u_{2}^{\beta}\right) \\
-\left(4.9 v_{1}^{M}, 0.5 v_{1}^{M}+4.4 v_{1}^{\alpha}, 0.5 v_{1}^{M}+5.4 v_{1}^{\beta}\right)-\left(2.6 v_{2}^{M}, 0.4 v_{2}^{M}+2.2 v_{2}^{\alpha}, 0.4 v_{2}^{M}+3 v_{2}^{\beta}\right)
\end{array}\right) \leq R(0,0,0), \\
& R\left(\begin{array}{c}
\left(2.5 u_{1}^{M}, 0.2 u_{1}^{M}+2.3 u_{1}^{\alpha}, 0.4 u_{1}^{M}+2.9 u_{1}^{\beta}\right)+\left(5.7 u_{2}^{M}, 0.2 u_{2}^{M}+5.5 u_{2}^{\alpha}, 0.2 u_{2}^{M}+5.9 u_{2}^{\beta}\right) \\
-\left(4.1 v_{1}^{M}, 0.7 v_{1}^{M}+3.4 v_{1}^{\alpha}, 0.7 v_{1}^{M}+4.8 v_{1}^{\beta}\right)-\left(2.3 v_{2}^{M}, 0.1 v_{2}^{M}+2.2 v_{2}^{\alpha}, 0.1 v_{2}^{M}+2.4 v_{2}^{\beta}\right)
\end{array}\right) \leq R(0,0,0), \\
& R\left(\begin{array}{c}
\left(5.1 u_{1}^{M}, 0.7 u_{1}^{M}+4.4 u_{1}^{\alpha}, 0.7 u_{1}^{M}+5.8 u_{1}^{\beta}\right)+\left(7.4 u_{2}^{M}, 0.9 u_{2}^{M}+6.5 u_{2}^{\alpha}, 0.9 u_{2}^{M}+8.3 u_{2}^{\beta}\right) \\
-\left(6.1 v_{1}^{M}, 0.2 v_{1}^{M}+5.9 v_{1}^{\alpha}, v_{1}^{M}+7.1 v_{1}^{\beta}\right)-\left(4.1 v_{2}^{M}, 0.5 v_{2}^{M}+3.6 v_{2}^{\alpha}, 0.5 v_{2}^{M}+4.6 v_{2}^{\beta}\right)
\end{array}\right) \leq R(0,0,0), \\
& R\left(u_{r}^{M}, u_{r}^{\alpha}, u_{r}^{\beta}\right) \geq R(0,0,0), \quad \forall r \\
& R\left(v_{i}^{M}, v_{i}^{\alpha}, v_{i}^{\beta}\right) \geq R(0,0,0) \quad \forall i \\
& u_{r}^{M}-u_{r}^{\alpha} \geq 0, \quad \forall r, \\
& v_{i}^{M}-v_{i}^{\alpha} \geq 0, \quad \forall i, \\
& u_{r}^{\alpha} \geq 0, u_{r}^{\beta} \geq 0, \quad \forall r \\
& v_{i}^{\alpha} \geq 0, v_{i}^{\beta} \geq 0, \quad \forall i .
\end{aligned}
$$

Step 4: Based on Definition 12, convert the above model to the following model:

$$
\begin{aligned}
& \max \theta_{A}=\left(\left(2.6 u_{1}^{M}-0.6 u_{1}^{\alpha}+0.7 u_{1}^{\beta}\right)+\left(4.1 u_{2}^{M}-0.95 u_{2}^{\alpha}+1.1 u_{2}^{\beta}\right)\right) \\
& \text { s.t. } \\
& \left(\left(4 v_{1}^{M}-0.875 v_{1}^{\alpha}+1.125 v_{1}^{\beta}\right)+\left(2.1 v_{2}^{M}-0.475 v_{2}^{\alpha}+0.575 v_{2}^{\beta}\right)\right)=1, \\
& \left(2.6 u_{1}^{M}-0.6 u_{1}^{\alpha}+0.7 u_{1}^{\beta}\right)+\left(4.1 u_{2}^{M}-0.95 u_{2}^{\alpha}+1.1 u_{2}^{\beta}\right)- \\
& \left(\left(4 v_{1}^{M}-0.875 v_{1}^{\alpha}+1.125 v_{1}^{\beta}\right)+\left(2.1 v_{2}^{M}-0.475 v_{2}^{\alpha}+0.575 v_{2}^{\beta}\right)\right) \leq 0, \\
& \left(2.2 u_{1}^{M}-0.55 u_{1}^{\alpha}+0.55 u_{1}^{\beta}\right)+\left(3.5 u_{2}^{M}-0.825 u_{2}^{\alpha}+0.925 u_{2}^{\beta}\right)- \\
& \left(\left(2.9 v_{1}^{M}-0.725 v_{1}^{\alpha}+0.725 v_{1}^{\beta}\right)+\left(1.5 v_{2}^{M}-0.35 v_{2}^{\alpha}+0.4 v_{2}^{\beta}\right)\right) \leq 0, \\
& \left(3.2 u_{1}^{M}-0.675 u_{1}^{\alpha}+0.925 u_{1}^{\beta}\right)+\left(5.1 u_{2}^{M}-1.075 u_{2}^{\alpha}+1.475 u_{2}^{\beta}\right)- \\
& \left(\left(4.9 v_{1}^{M}-1.1 v_{1}^{\alpha}+1.35 v_{1}^{\beta}\right)+\left(2.6 v_{2}^{M}-0.55 v_{2}^{\alpha}+0.75 v_{2}^{\beta}\right)\right) \leq 0, \\
& \left(2.55 u_{1}^{M}-0.575 u_{1}^{\alpha}+0.725 u_{1}^{\beta}\right)+\left(5.7 u_{2}^{M}-1.2 u_{2}^{\alpha}+1.65 u_{2}^{\beta}\right)- \\
& \left(\left(4.1 v_{1}^{M}-0.85 v_{1}^{\alpha}+1.2 v_{1}^{\beta}\right)+\left(2.3 v_{2}^{M}-0.55 v_{2}^{\alpha}+0.6 v_{2}^{\beta}\right)\right) \leq 0, \\
& \left(5.1 u_{1}^{M}-1.1 u_{1}^{\alpha}+1.45 u_{1}^{\beta}\right)+\left(7.4 u_{2}^{M}-1.625 u_{2}^{\alpha}+2.075 u_{2}^{\beta}\right)- \\
& \left(\left(6.3 v_{1}^{M}-1.475 v_{1}^{\alpha}+1.775 v_{1}^{\beta}\right)+\left(4.1 v_{2}^{M}-0.9 v_{2}^{\alpha}+1.15 v_{2}^{\beta}\right)\right) \leq 0, \\
& u_{1}^{M}-0.25 u_{1}^{\alpha}+0.25 u_{1}^{\beta} \geq 0, u_{2}^{M}-0.25 u_{2}^{\alpha}+0.25 u_{2}^{\beta} \geq 0, \\
& v_{1}^{M}-0.25 v_{1}^{\alpha}+0.25 v_{1}^{\beta} \geq 0, v_{2}^{M}-0.25 v_{2}^{\alpha}+0.25 v_{2}^{\beta} \geq 0, \\
& u_{1}^{M}-u_{1}^{\alpha} \geq 0, u_{2}^{M}-u_{2}^{\beta} \geq 0, \\
& v_{1}^{M}-v_{1}^{\alpha} \geq 0, v_{2}^{M}-v_{2}^{\alpha} \geq 0, \\
& u_{r}^{\alpha} \geq 0, u_{r}^{\beta} \geq 0, \quad r=1,2, \\
& v_{i}^{\alpha} \geq 0, v_{i}^{\beta} \geq 0, \quad i=1,2 .
\end{aligned}
$$


After computations with Lingo, we have the following optimal information for $\mathrm{DMU}_{\mathrm{A}}$ :

$u_{1}^{*}=(0.08682,0.08682,0.36267), u_{2}^{*}=(0.107402,0,0), v_{1}^{*}=(0.15182,0,0), v_{2}^{*}=(0.132123,0,0.20046)$.

And,

$$
\widetilde{\theta}_{A}^{*}=\left(\widetilde{\theta}_{1}^{M}, \widetilde{\theta}_{1}^{\alpha}, \widetilde{\theta}_{1}^{\beta}\right)=(0.6660,0.2579,1.0651) .
$$

Similarly, for other DMUs, we report the results in Table 4.

Table 4. Fuzzy efficiencies of the other DMUs.

\begin{tabular}{ccccc}
\hline & $\mathbf{D M U}_{\mathbf{B}}$ & $\mathbf{D M U}_{\mathbf{C}}$ & $\mathbf{D M U}_{\mathbf{D}}$ & $\mathbf{D M U}_{\mathbf{E}}$ \\
\hline$u_{1}^{*}$ & $(0,0,0)$ & $(0,0,1.08108)$ & $(0,0,0)$ & $(0,0,0.68965)$ \\
$u_{2}^{*}$ & $(0,0,1.08108)$ & $(0,0,0)$ & $(0.17544,0,0)$ & $(0,0,0)$ \\
$v_{1}^{*}$ & $(0,0,1.158997)$ & $(0,0,0.45747)$ & $(0.24390,0,0)$ & $(0.16949,0,0)$ \\
$v_{2}^{*}$ & $(0,0,0.39932)$ & $(0.14282,0,0.14783)$ & $(0,0,0)$ & $(0,0,0)$ \\
$\widetilde{\theta}^{*}$ & $(0,0,4)$ & $(0,0,4)$ & $(1,0.1579,0.1579)$ & $(0,0,4)$ \\
\hline
\end{tabular}

By these results, we can see that $\mathrm{DMU}_{\mathrm{A}}$ is inefficient, and the others are efficient. Further, we have used Wang et al.'s model [28] for comparing and ranking fuzzy efficiencies.

From Table 5, the DMUs are fully ranked in terms of their fuzzy efficiencies as follows:

$$
B=C=E \stackrel{60.57 \%}{\geq} D \stackrel{56.92 \%}{\geq} A .
$$

Table 5. The matrix of the degree of preference for fuzzy efficiencies obtained by model [28].

\begin{tabular}{cccccc}
\hline DMUs & $\boldsymbol{A}$ & $\boldsymbol{B}$ & $\boldsymbol{C}$ & $\boldsymbol{D}$ & $\boldsymbol{E}$ \\
\hline A & - & 0.4308 & 0.4308 & 0.3943 & 0.4308 \\
B & 0.5692 & - & 0.5 & 0.5557 & 0.5 \\
C & 0.5692 & 0.5 & - & 0.5557 & 0.5 \\
D & 0.6257 & 0.4443 & 0.4443 & - & 0.4443 \\
E & 0.5692 & 0.5 & 0.5 & 0.5557 & - \\
\hline
\end{tabular}

According to our model, $\mathrm{DMU}_{\mathrm{B}}, \mathrm{DMU}_{\mathrm{C}}$ and $\mathrm{DMU}_{\mathrm{E}}$ are efficient. However, by the model of [48], we can see that $\mathrm{DMU}_{\mathrm{B}}, \mathrm{DMU}_{\mathrm{D}}$ and $\mathrm{DMU} \mathrm{E}_{\mathrm{E}}$ are efficient. Even if the results were the same, because the model of [48] uses the wrong strategy, it would still not be valid. We take note that this example is utilized to demonstrate the computational procedure of the proposed technique and such a comparison is insignificant. Although the suggested procedure has been employed to a numerical case, the same frames could be used, with some adjustment, to other benchmarking problems.

\section{Conclusions and Future Work}

Within the past few years, a developing interest has appeared in fully fuzzy DEA (FFDEA) and presently there are numerous strategies to solve it. However, in original fuzzy sets, the certainty of the information is approximately ignored. Z-number is a suitable measure for comprehensive explanation of real-life information and has that extra ability of being able to depict uncertain information. This paper explains the drawbacks of Sotoudeh-Anvari et al.'s method [48] in the fully fuzzy DEA to rank DMUs with imprecise data. We show that the mentioned method did not consider the fuzzy axioms, so it may produce incorrect rankings in some cases. To remove the existing drawbacks, we presented a new fully fuzzified DEA, where all decision parameters and variables are Z-numbers. Based on our results, we can see that Sotoudeh-Anvari et al.'s method [48] should not be used for evaluating the best relative fuzzy efficiencies of DMUs. Furthermore, our model used Wang et al.'s model [28] for 
comparing and ranking fuzzy efficiencies. It is explained that our model provides the right evaluation of the relative efficiency of a DMU under ambiguous circumstances and gives more reliable results. In spite of the fact that the model, arithmetic operations and results introduced here have exhibited the viability of our approach, it might be additionally applied in other fuzzy DEA problem such as network FFDEA, FFDEA with common weights, and FFDEA with undesirable outputs. For future work, we plan to consider these issues.

Author Contributions: A.N. and S.E.N. contributed to generating the research ideas, designed the research, analyzed the data and developed the paper. M.F. and M.J. provided extensive advice throughout the study regarding the research design, methodology, and revised the manuscript. All the authors have read and approved the final manuscript.

Funding: This research received no external funding.

Acknowledgments: The authors would like to thank all the three reviewers and the editor for their valuable suggestions which have led to an improvement in both the quality and clarity of this paper.

Conflicts of Interest: The authors declare no conflict of interest.

\section{References}

1. Charnes, A.; Cooper, W.W.; Rhodes, E. Measuring the efficiency of decision making units. Eur. J. Oper. Res. 1978, 2, 429-444. [CrossRef]

2. Banker, R.D.; Charnes, A.; Cooper, W.W. Some models for estimating technical and scale inefficiencies in data envelopment analysis. Manag. Sci. 1984, 30, 1078-1092. [CrossRef]

3. Cooper, W.W.; Park, K.S.; Yu, G. IDEA and AR-IDEA: Models for dealing with imprecise data in DEA. Manag. Sci. 1999, 45, 597-607. [CrossRef]

4. Despotis, D.K.; Smirlis, Y.G. Data envelopment analysis with imprecise data. Eur. J. Oper. Res. 2002, 140, 24-36. [CrossRef]

5. Kao, C. Interval efficiency measures in data envelopment analysis with imprecise data. Eur. J. Oper. Res. 2006, 174, 1087-1099. [CrossRef]

6. Emrouznejad, A.; Parker, B.R.; Tavares, G. Evaluation of research in efficiency and productivity: A survey and analysis of the first 30 years of scholarly literature in DEA. Socio-Econ. Plan. Sci. 2008, 42, 151-157. [CrossRef]

7. Jahanshahloo, G.R.; Abbasian-Naghneh, S. Data envelopment analysis with imprecise data. Appl. Math. Sci. 2011, 5, 3089-3106.

8. Liu, J.S.; Lu, L.Y.; Lu, W.M. Research fronts in data envelopment analysis. Omega 2016, 58, 33-45. [CrossRef]

9. Wei, G.; Wang, J. A comparative study of robust efficiency analysis and data envelopment analysis with imprecise data. Expert Syst. Appl. 2017, 81, 28-38. [CrossRef]

10. Chen, Y.; Cook, W.D.; Du, J.; Hu, H.; Zhu, J. Bounded and discrete data and Likert scales in data envelopment analysis: Application to regional energy efficiency in China. Ann. Oper. Res. 2017, 255, 347-366. [CrossRef]

11. Toloo, M.; Keshavarz, E.; Hatami-Marbini, A. Dual-role factors for imprecise data envelopment analysis. Omega 2018, 77, 15-31. [CrossRef]

12. Zhou, X.; Xu, Z.; Yao, L.; Tu, Y.; Lev, B.; Pedrycz, W. A novel Data Envelopment Analysis model for evaluating industrial production and environmental management system. J. Clean. Prod. 2018, 170, 773-788. [CrossRef]

13. Zadeh, L.A. Fuzzy sets. Inf. Control 1965, 8, 338-353. [CrossRef]

14. Bellman, R.E.; Zadeh, L.A. Decision-making in a fuzzy environment. Manag. Sci. 1970, 17, 141-164. [CrossRef]

15. Tanaka, H.; Asai, K. A formulation of fuzzy linear programming based on comparison of fuzzy number. Control Cybern. 1984, 13, 185-194.

16. Campos, L.; Verdegay, J.L. Linear programming problems and ranking of fuzzy numbers. Fuzzy Sets Syst. 1989, 32, 1-11. [CrossRef]

17. Zimmermann, H.J. Fuzzy Set Theory—And Its Applications; Springer Science Business Media: Berlin, Germany, 2011.

18. Edalatpanah, S.A.; Shahabi, S. A new two-phase method for the fuzzy primal simplex algorithm. Int. Rev. Pure Appl. Math. 2012, 8, 157-164. 
19. Saberi Najafi, H.; Edalatpanah, S.A.; Dutta, H. A nonlinear model for fully fuzzy linear programming with fully unrestricted variables and parameters. Alex. Eng. J. 2016, 55, 2589-2595. [CrossRef]

20. Saberi Najafi, S.; Edalatpanah, S.A. A Note on A new method for solving fully fuzzy linear programming problems. Appl. Math. Model. 2013, 37, 7865-7867. [CrossRef]

21. Rodríguez, R.M.; Martínez, L.; Herrera, F.; Torra, V. A Review of Hesitant Fuzzy Sets: Quantitative and Qualitative Extensions. In Fuzzy Logic in Its 50th Year; Springer International Publishing: Cham, Switzerland, 2016; pp. 109-128.

22. Das, S.K.; Edalatpanah, S.A.; Mandal, T. A proposed model for solving fuzzy linear fractional programming problem: Numerical Point of View. J. Comput. Sci. 2018, 25, 367-375. [CrossRef]

23. Sengupta, J.K. A fuzzy systems approach in data envelopment analysis. Comput. Math. Appl. 1992, 24, 259-266. [CrossRef]

24. Triantis, K.; Girod, O. A mathematical programming approach for measuring technical efficiency in a fuzzy environment. J. Prod. Anal. 1998, 10, 85-102. [CrossRef]

25. Kao, C.; Liu, S.T. Fuzzy efficiency measures in data envelopment analysis. Fuzzy Sets Syst. 2000, 113, 427-437. [CrossRef]

26. Dia, M. A model of fuzzy data envelopment analysis. Inf. Syst. Oper. Res. 2004, 42, 267-279. [CrossRef]

27. Garcia, P.A.A.; Schirru, R.; Melo, P.F.F.E. A fuzzy data envelopment analysis approach for FMEA. Prog. Nucl. Energy 2005, 46, 359-373. [CrossRef]

28. Wang, Y.M.; Luo, Y.; Liang, L. Fuzzy data envelopment analysis based upon fuzzy arithmetic with an application to performance assessment of manufacturing enterprises. Expert Syst. Appl. 2009, 36, 5205-5211. [CrossRef]

29. Wang, Y.M.; Chin, K.S. Fuzzy data envelopment analysis: A fuzzy expected value approach. Expert Syst. Appl. 2011, 38, 11678-11685. [CrossRef]

30. Emrouznejad, A.; Tavana, M.; Hatami-Marbini, A. The state of the art in fuzzy data envelopment analysis. In Performance Measurement with Fuzzy Data Envelopment Analysis; Springer: Berlin/Heidelberg, Germany, 2014; pp. 1-45.

31. Puri, J.; Yadav, S.P. A fuzzy DEA model with undesirable fuzzy outputs and its application to the banking sector in India. Expert Syst. Appl. 2014, 41, 6419-6432. [CrossRef]

32. Wanke, P.; Barros, C.P.; Emrouznejad, A. Assessing productive efficiency of banks using integrated Fuzzy-DEA and bootstrapping: A case of Mozambican banks. Eur. J. Oper. Res. 2016, 249, 378-389. [CrossRef]

33. Hatami-Marbini, A.; Agrell, P.J.; Tavana, M.; Khoshnevis, P. A flexible cross-efficiency fuzzy data envelopment analysis model for sustainable sourcing. J. Clean. Prod. 2017, 142, 2761-2779. [CrossRef]

34. Nastis, S.A.; Bournaris, T.; Karpouzos, D. Fuzzy data envelopment analysis of organic farms. Oper. Res. 2017, 1-14. [CrossRef]

35. Lio, W.; Liu, B. Uncertain data envelopment analysis with imprecisely observed inputs and outputs. Fuzzy Optim. Decis. Mak. 2017, 17, 357-373. [CrossRef]

36. Hu, C.F.; Liu, F.B. Data envelopment analysis with non-LR type fuzzy data. Soft Comput. 2017, 21, 5851-5857. [CrossRef]

37. Hu, C.K.; Liu, F.B.; Hu, C.F. Efficiency measures in fuzzy data envelopment analysis with common weights. J. Ind. Manag. Optim. 2017, 13, 237-249. [CrossRef]

38. Izadikhah, M.; Khoshroo, A. Energy management in crop production using a novel Fuzzy Data Envelopment Analysis model. RAIRO-Oper. Res. 2018, 52, 595-617. [CrossRef]

39. Amirkhan, M.; Didehkhani, H.; Khalili-Damghani, K.; Hafezalkotob, A. Mixed uncertainties in data envelopment analysis: A fuzzy-robust approach. Expert Syst. Appl. 2018, 103, 218-237. [CrossRef]

40. Rezaee, M.J.; Jozmaleki, M.; Valipour, M. Integrating dynamic fuzzy C-means, data envelopment analysis and artificial neural network to online prediction performance of companies in stock exchange. Phys. A Stat. Mech. Appl. 2018, 489, 78-93. [CrossRef]

41. Wang, S.; Yu, H.; Song, M. Assessing the efficiency of environmental regulations of large-scale enterprises based on extended fuzzy data envelopment analysis. Ind. Manag. Data Syst. 2018, 118, 463-479. [CrossRef]

42. Mu, W.; Kanellopoulos, A.; van Middelaar, C.E.; Stilmant, D.; Bloemhof, J.M. Assessing the impact of uncertainty on benchmarking the eco-efficiency of dairy farming using fuzzy data envelopment analysis. J. Clean. Prod. 2018, 189, 709-717. [CrossRef] 
43. Zhou, X.; Xu, Z.; Chai, J.; Yao, L.; Wang, S.; Lev, B. Efficiency evaluation for banking systems under uncertainty: A multi-period three-stage DEA model. Omega 2018, in press. [CrossRef]

44. Hatami-Marbini, A.; Tavana, M.; Ebrahimi, A. A fully fuzzified data envelopment analysis model. Int. J. Inf. Decis. Sci. 2011, 3, 252-264.

45. Kazemi, M.; Alimi, A. A fully fuzzy approach to data envelopment analysis. J. Math. Comput. Sci. 2014, 11, 238-245. [CrossRef]

46. Puri, J.; Yadav, S.P. A fully fuzzy approach to DEA and multi-component DEA for measuring fuzzy technical efficiencies in the presence of undesirable outputs. Int. J. Syst. Assur. Eng. Manag. 2015, 6, 268-285. [CrossRef]

47. Khaleghi, S.; Noura, A.; Lotfi, F.H. Measuring Efficiency and Ranking Fully Fuzzy DEA. Indian J. Sci. Technol. 2015, 8. [CrossRef]

48. Sotoudeh-Anvari, A.; Najafi, E.; Sadi-Nezhad, S. A new data envelopment analysis in fully fuzzy environment on the base of the degree of certainty of information. J. Intell. Fuzzy Syst. 2016, 30, 3131-3142. [CrossRef]

49. Zadeh, L.A. A note on Z-numbers. Inf. Sci. 2011, 181, 2923-2932. [CrossRef]

50. Kang, B.; Wei, D.; Li, Y.; Deng, Y. A method of converting Z-number to classical fuzzy number. J. Inf. Comput. Sci. 2012, 9, 703-709.

51. Allahviranloo, T.; Hosseinzadeh Lotfi, F.; Kiasary, M.K.; Kiani, N.A.; Alizadeh, L. Solving fully fuzzy linear programming problem by the ranking function. Appl. Math. Sci. 2008, 2, 19-32.

52. Guo, P.; Tanaka, H. Fuzzy DEA: A perceptual evaluation method. Fuzzy Sets Syst. 2001, 119, 149-160. [CrossRef]

(C) 2018 by the authors. Licensee MDPI, Basel, Switzerland. This article is an open access article distributed under the terms and conditions of the Creative Commons Attribution (CC BY) license (http:/ / creativecommons.org/licenses/by/4.0/). 